\title{
Zinc Chloride Negatively Impacts Early Embryonic Development as Assessed using Human Embryonic Stem Cells as a Model ${ }^{\dagger}$
}

\author{
Girija Vasudevan ${ }^{1}$, Teresa D. Martinez ${ }^{1}$, Prue Talbot ${ }^{1}$ \\ 1 Department of Molecular, Cell and Systems Biology, University of California, Riverside, California \\ * Correspondence: endearing166@gmail.com; \\ $\dagger$ Presented at International e-Conference on Bioengineering for Health and Environment (ICBHE 2020)
}

Received: 5.07.2020; Revised: 10.07.2020; Accepted: 12.07.2020; Published: 15.07.2020

\begin{abstract}
Electronic cigarettes (ECs) or vaping products are nicotine delivery devices that have gained significant acceptance recently. EC or vaping product use associated lung injury (EVALI) has raised awareness regarding the damage caused by vaping. ECs expose the user and embryo/fetus to nicotine, flavor chemicals, solvents, metals, and reaction products. Little is known about how these chemicals affect prenatal development. Our prior work has shown that zinc is elevated in most EC aerosols. Our objective was to examine the effect of zinc on H9 human embryonic stem cell (hESC) colonies using live-cell imaging and to further determine if mitochondria were affected by zinc. hESC were plated, then treated with zinc chloride for 24 hours, after which the hESC were washed and labeled using Mitotracker deep red and imaged for 48 hours in a BioStation CT. StemCell QC was used to extract features from live-cell imaging data. We are using software such as MitoMo and CL Quant to examine the morphology of the mitochondria and analyze them. We observed that the mitochondria that were stressed would affect cell functionality and health. Stressed mitochondria will subsequently affect embryonic development; hence pregnant women should avoid using ECs during early pregnancy when the embryo is most susceptible to toxicants.
\end{abstract}

Keywords: Human Embryonic Stem Cells; Mitochondria; Zinc Chloride; Electronic Cigarettes;

Vaping.

(C) 2020 by the authors. This article is an open-access article distributed under the terms and conditions of the Creative Commons Attribution (CC BY) license (https://creativecommons.org/licenses/by/4.0/).

\section{Funding}

This research received no external funding.

\section{Acknowledgments}

This research has no acknowledgment.

\section{Conflicts of Interest}

The authors declare no conflict of interest. 